\begin{tabular}{|c|c|}
\hline Title & Theory of ultrabroadband optical pulse generation by induced phase modulation in a gas filled hollow waveguide \\
\hline Author(s) & Karasawa, Naoki; Morita, Ryuji; X u, Lin; Shigekawa, Hidemi; Y amashita, Mikio \\
\hline Citation & $\begin{array}{l}\text { Journal of the Optical Society of A merica B : Optical Physics, 16(4), 662-668 } \\
\text { https://doi.org/10.1364/JOSA B.16.000662 }\end{array}$ \\
\hline Issue Date & 1999-04-01 \\
\hline Doc URL & http:/hdl.handle.net/2115/45326 \\
\hline Rights & (c) 1999 Optical Society of A merica \\
\hline Type & article \\
\hline File Information & JOSA B16-4_662-668.pdf \\
\hline
\end{tabular}

Instructions for use 


\title{
Theory of ultrabroadband optical pulse generation by induced phase modulation in a gas-filled hollow waveguide
}

\author{
Naoki Karasawa, Ryuji Morita, and Lin Xu \\ Department of Applied Physics, Hokkaido University, and Core Research for Evolutional Science and Technology, \\ Japan Science and Technology Corporation, Kita-13, Nishi-8, Kita-Ku, Sapporo, 060-8628 Japan \\ Hidemi Shigekawa \\ Institute of Materials Science, and Center for Tsukuba Advanced Research Alliance, University of Tsukuba, \\ and Core Research for Evolutional Science and Technology, Japan Science and Technology Corporation, \\ 1-1-1 Tennodai, Tsukuba, 305-8573 Japan
}

Mikio Yamashita

Department of Applied Physics, Hokkaido University, and Core Research for Evolutional Science and Technology, Japan Science and Technology Corporation, Kita-13, Nishi-8, Kita-Ku, Sapporo, 060-8628 Japan

Received September 21, 1998; revised manuscript received December 24, 1998

\begin{abstract}
Generation of an ultrabroadband optical pulse with a fluent frequency dependency of the phase is important for creating a monocyclelike optical pulse and for shaping multiwavelength optical pulses. A previously proposed method that uses induced phase modulation between a femtosecond fundamental wave $\omega_{1}$ and its second-harmonic wave $\omega_{2}=2 \omega_{1}$ in a fused-silica fiber is applied to a capillary fiber filled with noble gas. Analytic results of chirps without dispersion but with loss in the fiber are shown, and the optimum conditions relating to a delay time between two pulses and to input peak powers are found for fully covering the spectrum between $\omega_{1}$ and $\omega_{2}$. Furthermore, numerical calculations, including dispersion effects of the fundamental and the second-harmonic waves from a Ti:sapphire laser-amplifier system with experimentally realizable parameters, are presented. These calculations show that it is possible to generate an ultrabroadband optical pulse whose spectrum ranges from 300 to $900 \mathrm{THz}(330$ to $1000 \mathrm{~nm})$ with quasi-linear chirp by this method. (C) 1999 Optical Society of America [S0740-3224(99)01604-5]
\end{abstract}

OCIS codes: $060.4370,060.7140,320.5520,320.5540,320.7140$.

\section{INTRODUCTION}

Recently, generation of an ultrabroadband optical pulse that uses induced phase modulation (IPM) in an optical glass fiber was proposed. ${ }^{1,2}$ In this method two or three optical pulses, one fundamental and others generated by second-harmonic generation or optical parametric amplification, were propagated inside a single-mode silica fiber at the same time; IPM, as well as self-phase modulation (SPM), among the pulses was used to broaden the spectrum of each pulse. Subsequently the phase of the combined pulses with quasi-linear chirp was compensated for by a spatial phase modulator. It was predicted that a monocyclelike optical pulse could be generated with this method.

Fused-silica fibers have conventionally been used to broaden the spectrum of an optical pulse through dispersive SPM, and one of the shortest pulses to date was generated by this method. ${ }^{3}$ Optical propagation loss of a fused-silica fiber is negligibly small for small distances. However, the damage threshold of fused-silica fiber is relatively small, which makes applications requiring large energies difficult. The maximum energy that can be allowed in this method is $\sim 40 \mathrm{~nJ}$ for a 13 -fs input pulse. ${ }^{4}$

In 1995 a high-powered, ultrabroadband continuum optical pulse was generated by multichannel propagation of a terawatt femtosecond near-infrared pulse in a long multimode capillary fiber filled with noble gas. ${ }^{5}$ However, the phases between frequency components of the generated optical wave were not constant in this method, since multichannel and multimode propagation was employed. More recently, single-mode capillary fibers filled with noble gases were used to broaden the spectrum of optical pulses through dispersive SPM in single-channel propagation, ${ }^{6,7}$ and sub-5-fs, high-powered pulses were generated with this technique. ${ }^{7}$ Because of the chemical stability of the noble gases, the damage threshold is relatively high, and the maximum energy that can be allowed is $\sim 10 \mu \mathrm{J}$. However, since the linear refractive index of the noble gas $(\simeq 1.0)$ is smaller than that of the glass used in the capillary wall, the optical field is not completely confined in the fiber during propagation. Hence loss al- 
ways occurs in this method. In addition the nonlinear refractive index and second-order dispersion of the noble gas are smaller than those of fused silica by a factor of thousands.

In this paper the analytic equations describing the chirp of each pulse in the case of two-pulse propagation in a single-mode capillary fiber are derived when dispersion is neglected. By use of these equations, effects of a delay time between two pulses (a femtosecond fundamental wave and its second-harmonic wave) for spectrum broadening are examined, and the optimum delay time and the input peak powers for fully covering the spectrum between two pulses are obtained. Furthermore, numerical calculations including second- and third-order dispersion terms are performed under experimentally feasible conditions. Since the second-harmonic wave as well as the fundamental wave are generated from one common femtosecond pulse, the carrier-phase difference between their waves is constant. ${ }^{8-11}$ This uniformity enables us to synthesize constructively the two spectrally broadened waves at the fiber output. A compressed pulse width is evaluated after its phase is compensated for by use of a spatial phase modulator.

\section{CHIRPS WITHOUT DISPERSION EFFECTS}

Nonlinear chirps are considered in the case in which dispersion effects can be neglected. For a capillary fiber with a $50-\mu \mathrm{m}$ radius filled with argon at $3.3 \mathrm{~atm}(2.5$ $\times 10^{3}$ torr) and at $300 \mathrm{~K}$, the dispersion length $L_{d}$ $=T_{0}{ }^{2} / \beta_{2}$ is $35.3 \mathrm{~m}$ for a fundamental wave $\left(\lambda_{1}=790\right.$ $\mathrm{nm}$, pulse 1) of a Ti:sapphire laser-amplifier system when its full width at half-maximum (FWHM, $T_{\mathrm{FWHM}}$ ) is $30 \mathrm{fs}$ $\left[T_{0}=T_{\text {FWHM }} /(2 \sqrt{\ln 2})\right.$ for a Gaussian pulse $]$. For a second-harmonic wave $\left(\lambda_{2}=395 \mathrm{~nm}, \quad T_{\mathrm{FWHM}}=30 \mathrm{fs}\right.$, pulse 2), it is $2.46 \mathrm{~m}$. As will be shown below, we consider mainly a short fiber length of $\sim 0.3 \mathrm{~m}$. Thus, for this fiber length, it can be estimated that the dispersion effect is small and not very significant. Also, since the experimentally observed spectra ${ }^{6,7}$ of an argon-filled fiber taken under conditions similar to those in this paper do not show significant asymmetry owing to self-steepening effects, we neglect these terms in the following analysis.

We consider a case in which two optical pulses with different center frequencies and widths are copropagating in a capillary fiber with a length $z_{l}$ whose axis is parallel to the $z$ direction. For each pulse $i(i=1,2)$ the electric field (linearly polarized in the $x$ direction) can be written as follows:

$$
\begin{aligned}
\mathbf{E}_{i}\left(\mathbf{r}_{i}, t\right)= & 1 / 2 \hat{\mathbf{x}}\left\{F_{i}(x, y) A_{i}(z, t)\right. \\
& \left.\times \exp \left[i\left(\beta_{0 i} z-\omega_{i} t+\phi_{0 i}\right)\right]+\text { c.c. }\right\},
\end{aligned}
$$

where $\beta_{0 i}, \omega_{i}$, and $\phi_{0 i}$ are the wave number of the propagation mode, the center angular frequency, and the constant phase for a pulse $i$ and c.c. represents the complex conjugate. $F_{i}(x, y)$ represents the transverse distribution of the mode, and $A_{i}(z, t)$ represents the slowly varying envelope part of the field. For convenience two timecoordinates that move with the speed of a group velocity for each pulse are defined as $T_{i}=t-\beta_{1 i} z$, where $\beta_{1 i}$ is the inverse of the group velocity of pulse $i$. By use of a slowly varying envelope approximation and if dispersion terms are neglected, $A_{i}\left(z, T_{i}\right)$ satisfies the following equations $^{12,13}$ :

$$
\begin{aligned}
& \frac{\partial A_{1}}{\partial z}=-\frac{\alpha_{1}}{2} A_{1}+i \frac{n_{2} \omega_{1}}{c}\left[f_{11}\left|A_{1}\right|^{2}+2 f_{12}\left|A_{2}\right|^{2}\right] A_{1}, \\
& \frac{\partial A_{2}}{\partial z}=-\frac{\alpha_{2}}{2} A_{2}+i \frac{n_{2} \omega_{2}}{c}\left[f_{22}\left|A_{2}\right|^{2}+2 f_{12}\left|A_{1}\right|^{2}\right] A_{2},
\end{aligned}
$$

where $\alpha_{i}$ represents the loss, $n_{2}$ is the nonlinear refractive index, and $c$ is the speed of light. $f_{i j}$ is the mode overlap integral between transverse modes of pulses $i$ and $j$. Below these values are all assumed to be the inverse of the core area of the fiber. The relative phase difference between pulses $\left(\phi_{01}-\phi_{02}\right)$ does not enter into Eqs. (2) and (3). Thus the spectrum broadening due to IPM does not depend on phase difference. However, it has some effects when two pulses are combined for pulse compression as discussed in Section 6. With

$$
A_{i}=\sqrt{P_{i}} \exp \left(-\frac{\alpha_{i} z}{2}\right) U_{i}\left(z, T_{i}\right),
$$

where $P_{i}$ is the peak power of pulse $i$, the solutions of Eqs. (2) and (3) are given by

$$
U_{i}\left(z, T_{i}\right)=\exp \left[i \phi_{i}\left(z, T_{i}\right)\right] U_{i}\left(0, T_{i}\right) .
$$

Here the nonlinearly time-dependent phases $\phi_{i}$ are given by

$$
\begin{aligned}
\phi_{1}\left(z_{l}, T_{1}\right)= & \frac{n_{2} \omega_{1}}{c}\left[f_{11} P_{1}\left|U_{1}\right|^{2} z_{1 \text { eff }}+2 f_{12} P_{2}\right. \\
& \left.\times \int_{0}^{z_{l}} \exp \left(-\alpha_{2} x\right)\left|U_{2}\left(0, T_{1}-x d\right)\right|^{2} \mathrm{~d} x\right], \\
\phi_{2}\left(z_{l}, T_{2}\right)= & \frac{n_{2} \omega_{2}}{c}\left[f_{22} P_{2}\left|U_{2}\right|^{2} z_{2 \text { eff }}+2 f_{12} P_{1}\right. \\
& \left.\times \int_{0}^{z_{l}} \exp \left(-\alpha_{1} x\right)\left|U_{1}\left(0, T_{2}+x d\right)\right|^{2} \mathrm{~d} x\right],
\end{aligned}
$$

where $d$ is the difference between the inverses of the group velocities,

$$
d=\beta_{12}-\beta_{11} .
$$

The first terms on the right-hand sides of Eqs. (4) and (5) show the time-dependent phase that is due to SPM. Because of the loss, fiber length $z_{l}$ is reduced to effective length $z_{i \text { eff }}$ :

$$
z_{i \text { eff }}=\left[1-\exp \left(-\alpha_{i} z_{l}\right)\right] / \alpha_{i} .
$$

The nonlinear chirp, which represents the instantaneous frequency shift from the carrier frequency that is due to the nonlinearly time-dependent phase, can be calculated to be 


$$
\delta \omega_{i}=-\frac{\partial \phi_{i}}{\partial T_{i}},
$$

where the minus signs arise from the definition of the electric field in Eq. (1). These quantities can be calculated if the pulse shapes are specified. In this section, to obtain the analytic formula, we assume the following Gaussian input-pulses:

$$
\begin{aligned}
& A_{1}\left(0, T_{1}\right)=\sqrt{P_{1}} \exp \left[-T_{1}^{2} /\left(2 T_{01}^{2}\right)\right] \\
& A_{2}\left(0, T_{2}\right)=\sqrt{P_{2}} \exp \left[-\left(T_{2}-T_{d 2}\right)^{2} /\left(2 T_{02}^{2}\right)\right] ;
\end{aligned}
$$

$T_{0 i}$ is the pulse width, where the intensity becomes $1 / e$, and $T_{d 2}$ is the delay time of pulse 2 with respect to pulse 1. The chirps are calculated to be

$$
\begin{aligned}
\delta \omega_{1}\left(z_{l},\right. & \left.T_{1}\right) \\
= & \frac{2 n_{2} \omega_{1}}{c} \frac{z_{l}}{T_{01}}\left(\frac{z_{1 \text { eff }}}{z_{l}} f_{11} \tau_{1} \exp \left(-\tau_{1}^{2}\right) P_{1}+\frac{f_{12}}{\delta_{1}}\right. \\
& \times \exp \left[-2 \eta_{2}\left(\tau_{2}-\tau_{d 2}\right)+\eta_{2}^{2}\right] P_{2} \sqrt{\pi} \eta_{2} \\
& \times\left\{\operatorname{erf}\left(\tau_{2}-\tau_{d 2}-\eta_{2}\right)-\operatorname{erf}\left(\tau_{2}-\tau_{d 2}-\eta_{2}-\delta_{2}\right)\right\} \\
& +\frac{f_{12}}{\delta_{1}} \exp \left[-2 \eta_{2}\left(\tau_{2}-\tau_{d 2}\right)+\eta_{2}{ }^{2}\right] P_{2} \\
& \times\left\{\exp \left[-\left(\tau_{2}-\tau_{d 2}-\eta_{2}-\delta_{2}\right)^{2}\right]\right. \\
& \left.\left.-\exp \left[-\left(\tau_{2}-\tau_{d 2}-\eta_{2}\right)^{2}\right]\right\}\right),
\end{aligned}
$$

$\delta \omega_{2}\left(z_{l}, T_{2}\right)$

$$
\begin{aligned}
= & \frac{2 n_{2} \omega_{2}}{c} \frac{z_{l}}{T_{02}}\left(\frac{z_{2 \text { eff }}}{z_{l}} f_{22}\left(\tau_{2}^{\prime}-\tau_{d 2}\right) \exp \left[-\left(\tau_{2}^{\prime}-\tau_{d 2}\right)^{2}\right] P_{2}\right. \\
& -\frac{f_{12}}{\delta_{2}} \exp \left(2 \eta_{1} \tau_{1}^{\prime}+\eta_{1}^{2}\right) P_{1} \sqrt{\pi} \eta_{1} \\
& \times\left\{\operatorname{erf}\left(\tau_{1}^{\prime}+\eta_{1}+\delta_{1}\right)-\operatorname{erf}\left(\tau_{1}^{\prime}+\eta_{1}\right)\right\} \\
& -\frac{f_{12}}{\delta_{2}} \exp \left(2 \eta_{1} \tau_{1}^{\prime}+\eta_{1}^{2}\right) P_{1}\left\{\exp \left[-\left(\tau_{1}^{\prime}+\eta_{1}+\delta_{1}\right)^{2}\right]\right. \\
& \left.\left.-\exp \left[-\left(\tau_{1}^{\prime}+\eta_{1}\right)^{2}\right]\right\}\right) .
\end{aligned}
$$

Here $\operatorname{erf}(x)=(2 / \sqrt{\pi}) \int_{0}^{x} \exp \left(-t^{2}\right) \mathrm{d} t$ is an error function, and the following quantities are defined as

$$
\begin{aligned}
\tau_{i} & =T_{1} / T_{0 i}, & \tau_{i}^{\prime} & =T_{2} / T_{0 i}, \quad \tau_{d 2}=T_{d 2} / T_{02}, \\
\delta_{i} & =z_{l} d / T_{0 i}, & \eta_{i} & =\alpha_{i} T_{0 i} / 2 d .
\end{aligned}
$$

By defining the walk-off length $L_{w i}$ as $L_{w i}$ $=T_{0 i} /|d|,^{12,13}$ we can write $\delta_{i}=\operatorname{sgn}(d) z_{l} / L_{w i}$ and $\eta_{i}$ $=\operatorname{sgn}(d) \alpha_{i} L_{w i} / 2$, where $\operatorname{sgn}(d)$ is \pm 1 according to the sign of $d$. Thus $\eta_{i}$ shows the estimate of the loss during the propagation of pulse $i$ for the walk-off length $L_{w i}$.

In Fig. 1 chirps are shown as functions of normalized time for the case in which $\omega_{1}<\omega_{2}$ and the group velocity of pulse 1 is greater than that of pulse $2(d>0$, in the normal dispersion region). For Fig. 1 the delay time $T_{d 2}$ is chosen such that two pulses meet near the fiber exit end. For $\omega_{1}$ [Fig. 1(a)] the pulse center is at time 0 , and for $\omega_{2}$ [Fig. 1(b)] the pulse center is at time $\tau_{d 2}$ $=-2.32$. Also, $T_{01}=T_{02}$ is assumed for this figure.

In Eqs. (6) and (7) the first terms on the right-hand sides are due to SPM, and the second and third terms are
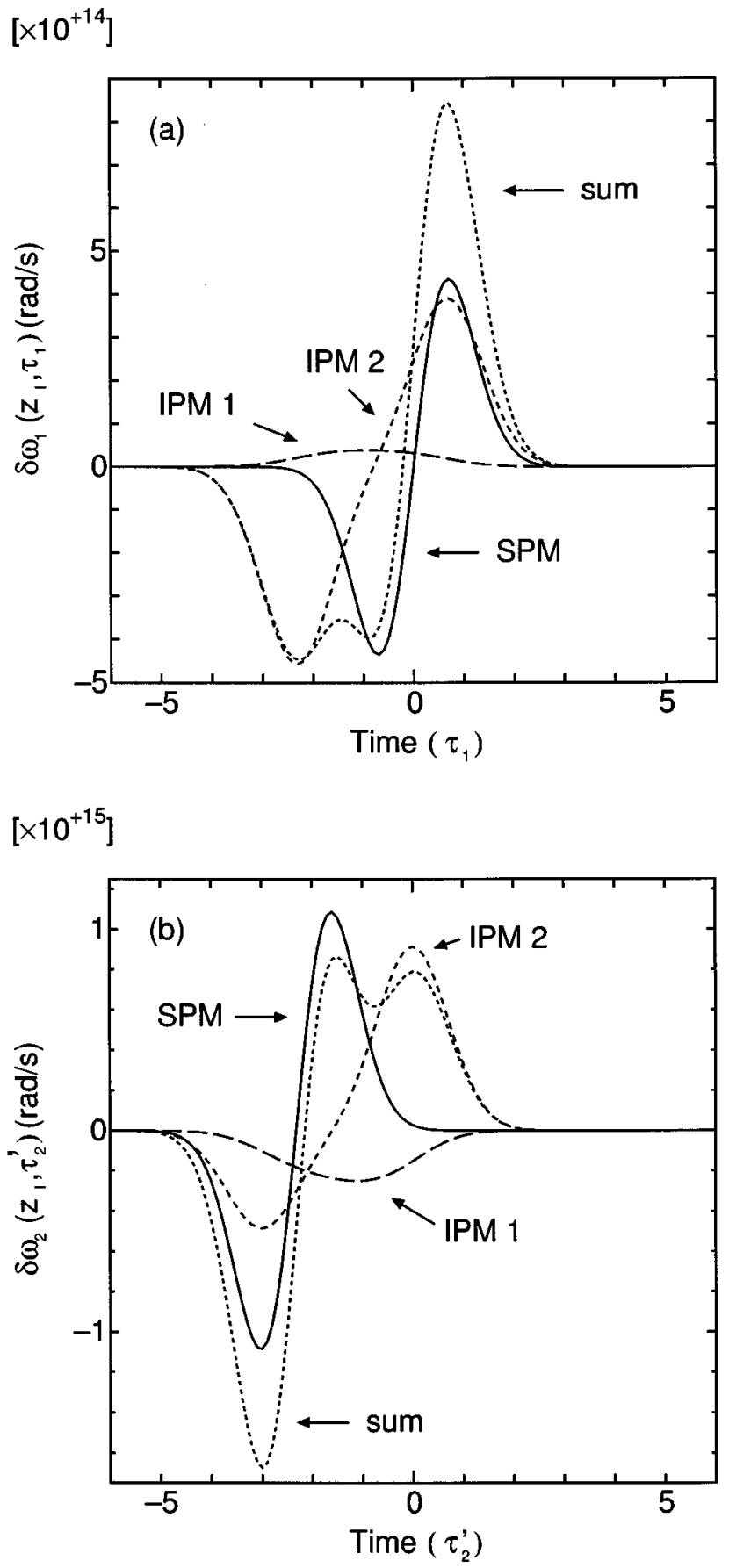

Fig. 1. Calculated chirps versus normalized time for (a) $\delta \omega_{1}$ and (b) $\delta \omega_{2}$ when dispersion terms are neglected. Solid curves show chirps from SPM, long- and short-dashed curves show chirps from IPM, and dotted curves show the total chirps. Pulse centers are at 0 for (a) and at -2.32 for (b). In calculations $\omega_{1}$ is the fundamental wave and $\omega_{2}$ is the second-harmonic wave of a Ti:sapphire laser system $\left(\lambda_{1}=790 \mathrm{~nm}\right)$. These are propagated in an argon-filled ( $300 \mathrm{~K}, 3.3 \mathrm{~atm})$ capillary fiber with a radius of $50 \mu \mathrm{m}$ and a length of $29.2 \mathrm{~cm}$. Also, $P_{1}=P_{2}=1.29 \mathrm{GW}, T_{01}$ $=T_{02}=30 /(2 \sqrt{\ln 2}) \mathrm{fs}\left(\tau_{1}=\tau_{2}, \quad \tau_{1}^{\prime}=\tau_{2}^{\prime}\right), n_{2}=3.234 \times 10^{-23}$ $\mathrm{m}^{2} / \mathrm{W}$, and $T_{d 2}=-41.8 \mathrm{fs}\left(T_{d 2} \simeq-z_{l} d\right)$ are used. 
due to IPM. Significantly, the second terms appear because of the loss during propagation.

Physically, these terms can be interpreted as follows. The intense electric field of the pulse causes an increase in the refractive index of the medium owing to the thirdorder optical nonlinearity, and its refractive-index change is proportional to the instantaneous intensity of the pulse. The refractive-index change causes nonlinearly timedependent change in the phase of the pulse. In the case of SPM, the temporal profile of this phase is proportional to the pulse shape. Chirp is the negative of the time derivative of this phase, which yields a lower frequency shift for the pulse leading edge and a higher frequency shift for the pulse trailing edge [solid curves (SPM) in Fig. 1]. In the case of IPM, the phase and the chirp depend on the group-velocity difference between pulses and on the fiber length. If a delay time is chosen such that $-z_{l} d<T_{d 2}$ $<0$ (pulse 2 leads), the center of pulse 1 passes the center of pulse 2 inside the fiber. For pulse 1, the effect of pulse 2 on the phase is larger at the leading edge than that at the trailing edge as pulse 1 passes pulse 2 , since the intensity of pulse 2 decreases, owing to the loss during propagation. This effect causes the higher frequency shift [long-dashed curve (IPM 1) in Fig. 1(a)]. On the other hand, for pulse 2 , the effect of pulse 1 on the phase becomes larger at the trailing edge than that at the leading edge as pulse 2 is passed by pulse 1, owing to the loss of pulse 1. This effect causes the lower frequency shift [long-dashed curve (IPM 1) in Fig. 1(b)]. The third terms of Eqs. (6) and (7) are caused mainly by the imperfect overlap between two pulses at one end of the fiber. For $T_{d 2} \simeq 0$ (when the pulses meet near the entrance of the fiber), the trailing edge of pulse 1 interacts with the leading edge of pulse 2. This interaction causes an increase of the phase only at the trailing edge of pulse 1 (the leading edge of pulse 2). Thus for pulse 1 (pulse 2), the phase is an increasing (decreasing) function with respect to time. Thus pulse 1 (pulse 2) is frequency-shifted lower (higher) near the center of the pulse. For $T_{d 2} \simeq-z_{l} d$ (when the pulses meet near the exit of the fiber), the leading edge of pulse 1 interacts with the trailing edge of pulse 2 , and pulse 1 is frequency-shifted higher near the center of the pulse [short-dashed curve (IPM 2) in Fig. 1(a)]. Also, pulse 2 is frequency-shifted lower near the center of the pulse [short-dashed curve (IPM 2) in Fig. 1(b)]. The total chirp for each wave is given by the sum of three terms in Eqs. (6) or (7), and its shape can be controlled by changing the delay time $T_{d 2}$, as is shown Section 3 .

\section{DETERMINATION OF A DELAY TIME FOR SPECTRUM BROADENING}

We set $\omega_{1}$ as the fundamental wave and $\omega_{2}=2 \omega_{1}$ as the second-harmonic wave. To cover the spectrum fully between $\omega_{1}$ and $\omega_{2}$, we desire to maximize $\delta \omega_{1}$ and $-\delta \omega_{2}$ $\left(\delta \omega_{2}<0\right)$. From Eq. (6) and Fig. 1 it can be seen that the first (SPM) term becomes maximum at $\tau_{1}=1 / \sqrt{2}$. For not very small $\delta_{2}$, the second term becomes maximum approximately at $\tau_{2}=\tau_{d 2}+\eta_{2}+\delta_{2} / 2$, and the third term becomes maximum approximately at $\tau_{2}=\tau_{d 2}$ $+\eta_{2}+\delta_{2}$. If $\sqrt{\pi} \eta_{2}<1$, i.e., the optical field does not become less than 1/e owing to the loss after the field propagates over the length $\sqrt{\pi} L_{w 2}$, we may ignore the second term. By use of $\tau_{2}=T_{01} \tau_{1} / T_{02}=T_{01} /\left(\sqrt{2} T_{02}\right)$ in the last equation and Eqs. (8) and (9), the optimum delay is given by

$$
T_{d 2}=T_{01} / \sqrt{2}-T_{02} \eta_{2}-z_{l} d .
$$

From Eq. (7), similar considerations give, for the condition of maximizing $-\delta \omega_{2}$,

$$
T_{d 2}=T_{02} / \sqrt{2}-T_{01} \eta_{1}-z_{l} d .
$$

A comparison of Eqs. (10) and (11) shows that, for small $\eta_{1}$ and $\eta_{2}$, the two conditions above can be satisfied if both pulses have the same widths $\left(T_{01}=T_{02}\right)$. In particular, if there are no losses $\left(\eta_{1}=\eta_{2}=0\right)$, these conditions can be satisfied exactly. The condition

$$
T_{d 2}=T_{02} / \sqrt{2}-z_{l} d
$$

specifies that both pulses meet almost at the fiber exit end.

\section{REQUIRED PEAK POWERS FOR SPECTRUM BROADENING}

Here we evaluate the pulse peak powers that can broaden the spectrum of each pulse such that spectra of these two pulses overlap. For this purpose we use the results of Section 3; i.e., the widths of both pulses are assumed to be equal, and the optimal delay time is given by Eq. (10). Also, loss terms in the chirp equations [the second terms on the right-hand sides in Eqs. (6) and (7)] are assumed to be small and can be ignored. However, since these terms give chirps in the favored directions (a higher frequency shift for $\delta \omega_{1}$ and a lower frequency shift for $\delta \omega_{2}$ ), the required peak powers obtained will be slightly larger than the practical experimental powers. Moreover, inspection of Eqs. (6) and (7) shows that the second and third terms become smaller than the first terms if $\delta_{1}$ or $\delta_{2}$ becomes larger than $\sim 3$. This characteristic implies that the fiber length must be smaller than the same value to have the effect of IPM comparable with that of SPM. Here we set the values of $\delta_{i}$ to be 3 , i.e., the fiber length $z_{l}$ is equal to $3 L_{w 1}\left(=3 L_{w 2}\right)$. Under these assumptions, the maximum chirps are given as follows:

$$
\begin{aligned}
\left(\delta \omega_{1}\right)_{\max }= & \frac{2 n_{2} \omega_{1}}{c \pi a^{2}} \frac{3}{d}\left\{0.4289 \frac{z_{1 \text { eff }}}{z_{l}} P_{1}\right. \\
& \left.+\frac{1}{3} \exp \left[-\eta_{2}\left(\eta_{2}+6\right)\right] P_{2}\right\}, \\
\left(-\delta \omega_{2}\right)_{\max }= & \frac{4 n_{2} \omega_{1}}{c \pi a^{2}} \frac{3}{d}\left\{0.4289 \frac{z_{2} \text { eff }}{z_{l}} P_{2}\right. \\
& \left.+\frac{1}{3} \exp \left[-\eta_{1}\left(\eta_{1}+6\right)\right] P_{1}\right\} .
\end{aligned}
$$

Here 0.4289 is the maximum of the SPM term $\tau_{i} \exp \left(-\tau_{i}^{2}\right.$ ), and $f_{i j}$ is replaced by $1 / \pi a^{2}$ ( $a$ is the capillary radius). For fully covering the spectrum between $\omega_{1}$ and $\omega_{2}$, we set the sum of Eqs. (13) and (14) to be $\omega_{1}$. This modification gives 


$$
\begin{aligned}
&\left\{0.4289 \frac{z_{1 \text { eff }}}{z_{l}}+\frac{2}{3} \exp \left[-\eta_{1}\left(\eta_{1}+6\right)\right]\right\} P_{1} \\
&+\left\{0.8578 \frac{z_{2 \text { eff }}}{z_{l}}+\frac{1}{3} \exp \left[-\eta_{2}\left(\eta_{2}+6\right)\right]\right\} P_{2} \\
&=\frac{c \pi a^{2}}{2 n_{2}} \frac{d}{3} .
\end{aligned}
$$

In Section 5 the practical values of $P_{1}$ and $P_{2}$ will be calculated for a capillary fiber filled with argon.

\section{ARGON CASE}

It is assumed that we employ an amplified Ti:sapphire laser system with a fundamental wavelength of $790 \mathrm{~nm}$ and a pulse width of $30 \mathrm{fs}$. The pulse width of the secondharmonic wave is assumed to be $30 \mathrm{fs}$ also. To calculate dispersion and loss characteristics of the fiber propagation mode in an argon-filled capillary fiber, we assume that the dominant propagation mode is $E H_{11}$, whose loss is the smallest. Then the phase constant $\beta$ and the field attenuation constant $\alpha / 2$ are given by ${ }^{14}$

$$
\begin{aligned}
& \beta=\frac{2 \pi}{\lambda}\left[1-\frac{1}{2}\left(\frac{2.405 \lambda}{2 \pi a}\right)^{2}\right], \\
& \frac{\alpha}{2}=\left(\frac{2.405}{2 \pi}\right)^{2} \frac{\lambda^{2}}{2 a^{3}} \frac{\nu^{2}+1}{\left(\nu^{2}-1\right)^{1 / 2}} .
\end{aligned}
$$

Here $\lambda$ is the wavelength in the gas medium; $\nu$ is the ratio of the linear refractive indices of the external medium to that of the internal media and is assumed to be 1.45. To obtain the group velocity, the group-velocity dispersion, and the third-order dispersion, it is necessary to calculate the derivatives of the phase constant with respect to the angular frequency. To include the effect of medium dispersion, pressure, and temperature, $\lambda$ in Eq. (16) is replaced by $2 \pi c /(\omega n)$, where the linear refractive index of gas $n$ depends on pressure $p(\mathrm{~atm} ; 1 \mathrm{~atm}=735.6$ torr $)$ and temperature $T(\mathrm{~K})$ as follows: ${ }^{15}$

$$
n=\left(2 \frac{n_{0}^{2}-1}{n_{0}^{2}+2} \frac{p T_{0}}{p_{0} T}+1\right)^{1 / 2}\left(1-\frac{n_{0}^{2}-1}{n_{0}^{2}+2} \frac{p T_{0}}{p_{0} T}\right)^{-1 / 2},
$$

where $n_{0}$ is the linear refractive index under standard conditions $\left(p_{0}=1 \mathrm{~atm}, T_{0}=273.15 \mathrm{~K}\right)$ and is given by the dispersion formula ${ }^{16}$

$$
\begin{aligned}
n_{0}{ }^{2}-1= & 5.547 \times 10^{-4}\left(1+\frac{5.15 \times 10^{5}}{\lambda_{0}{ }^{2}}+\frac{4.19 \times 10^{11}}{\lambda_{0}{ }^{4}}\right. \\
+ & \left.\frac{4.09 \times 10^{17}}{\lambda_{0}{ }^{6}}+\frac{4.32 \times 10^{23}}{\lambda_{0}{ }^{8}}\right),
\end{aligned}
$$

where $\lambda_{0}$ (in angstroms) is the vacuum wavelength.

For argon at $T=300 \mathrm{~K}, p=3.3 \mathrm{~atm}$, and $a$ $=50 \mu \mathrm{m}$, we obtain the following parameters $\left(n_{2}\right.$ from Ref. 6):

$$
\begin{aligned}
n_{2} & =3.234 \times 10^{-23} \mathrm{~m}^{2} / \mathrm{W}, \\
\lambda_{1} & =790 \mathrm{~nm}, \quad \lambda_{2}=395 \mathrm{~nm}, \\
\alpha_{1} / 2 & =1.0807 \mathrm{~m}^{-1}, \quad \alpha_{2} / 2=0.2702 \mathrm{~m}^{-1}, \\
\eta_{1} & =0.1051, \quad \eta_{2}=0.0263, \\
d & =\beta_{12}-\beta_{11} \\
& =3.338737 \times 10^{-09} \mathrm{~s} / \mathrm{m}-3.338552 \times 10^{-09} \mathrm{~s} / \mathrm{m} \\
& =1.85 \times 10^{-13} \mathrm{~s} / \mathrm{m} .
\end{aligned}
$$

To satisfy the condition $\delta_{i}=z_{l} d / T_{0 i}=3$, we need to have $z_{l}=29.2 \mathrm{~cm}$; for this fiber length the loss for the fundamental wave is $47 \%$ and that of the secondharmonic wave is $15 \%$. Also, $z_{1 \text { eff }}=21.7 \mathrm{~cm}$, and $z_{2 \text { eff }}$ $=27.0 \mathrm{~cm}$.

The optimal delay time from Eq. (10) is given by

$$
\begin{aligned}
T_{d 2} / T_{01} & =1 / \sqrt{2}-\delta_{i}-\eta_{2}=-2.3192, \\
T_{d 2} & =-41.8 \mathrm{fs} .
\end{aligned}
$$

From Eq. (15) the minimum powers required for fully covering the spectrum between the fundamental and the second-harmonic waves are given by

$$
0.298 P_{1}+0.480 P_{2}=1 \text {, }
$$

where $P_{i}$ is measured in gigawatts. From this equation, if we set $P_{1}=P_{2}$, the required minimum powers are $P_{1}$ $=P_{2}=1.29 \mathrm{GW}$.

\section{NUMERICAL CALCULATIONS}

To verify the above results with the dispersion terms, numerical calculations are performed. Here the program used in Ref. 1 is slightly modified to include the secondand the third-order dispersion terms of argon. The calculated values of the second- and the third-order dispersion terms $\left(\beta_{2 i}\right.$ and $\left.\beta_{3 i}\right)$ are as follows:

$$
\begin{array}{ll}
\beta_{21}=9.18 \mathrm{fs}^{2} / \mathrm{m}, & \beta_{22}=132 \mathrm{fs}^{2} / \mathrm{m}, \\
\beta_{31}=91.9 \mathrm{fs}^{3} / \mathrm{m}, & \beta_{32}=44.1 \mathrm{fs}^{3} / \mathrm{m} .
\end{array}
$$

Note that the second-order dispersion term for the fundamental wave $\left(9.18 \mathrm{fs}^{2} / \mathrm{m}\right)$ is much smaller than the value $\left(40.3 \mathrm{fs}^{2} / \mathrm{m}\right)$ for $a=80 \mu \mathrm{m}$ with the same pressure and temperature (cf. Ref. 7).

In the numerical calculations all the parameters shown in Section 5 are used except for peak powers and a delay time. Figure 2 shows the calculated spectrum with $T_{d 2}$ $=-41.8 \mathrm{fs}$ and $P_{1}=P_{2}=1.29 \mathrm{GW}$. Figure 3 shows the calculated spectrum with $T_{d 2}=0 \mathrm{fs}$ and $P_{1}=P_{2}$ $=1.29 \mathrm{GW}$. In these calculations the constant carrier phases are set to be $\phi_{01}=0$ and $\phi_{02}=-\pi / 2$. It was found that these values do not influence the spectrum except for the small interference part in which the highestfrequency edge of fundamental-wave spectrum and the lowest-frequency edge of the second-harmonic-wave spectrum overlap. ${ }^{2}$ Figure 4 shows the compressed pulse whose spectrum is shown in Fig. 2. This compression is performed by compensating for the phase of the fiberoutput composed pulse by the 256-channel spatial phase modulator according to Ref. 1 . The calculated pulse du- 
ration is $1.53 \mathrm{fs}$. In this method ${ }^{17}$ each spectral component of the output pulse from the fiber is projected to a different channel of the spatial phase modulator by a first spectral-spatial converter and a lens. Then the phase at each channel is adjusted independently to make it essentially the same at every channel for pulse compression. After this adjustment these spectral components are recombined by a second spectral-spatial converter and a lens to form a final pulse. Gratings have been used for spectral-spatial converters. However, these may not work for ultrabroadband pulses treated here owing to the overlap of different diffraction orders. ${ }^{18}$ Prisms can be used here, since added nonlinear material dispersion can be compensated for by the spatial phase modulator. ${ }^{19}$

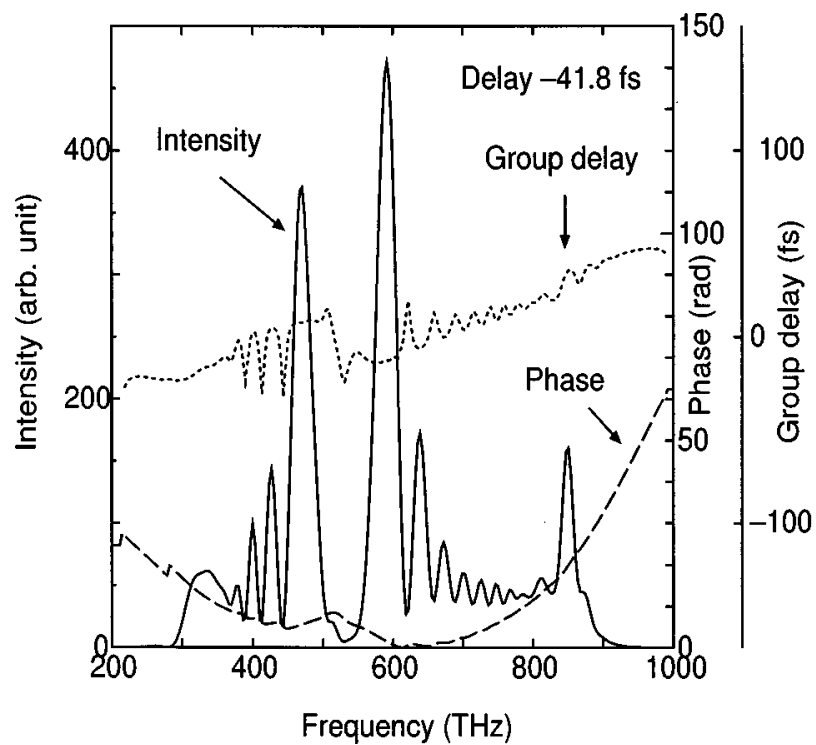

Fig. 2. Calculated intensity, phase, and group delay versus frequency of the fundamental and second-harmonic waves after propagation through an argon-filled capillary fiber under the condition $P_{1}=P_{2}=1.29 \mathrm{GW}, T_{d 2}=-41.8 \mathrm{fs}$.

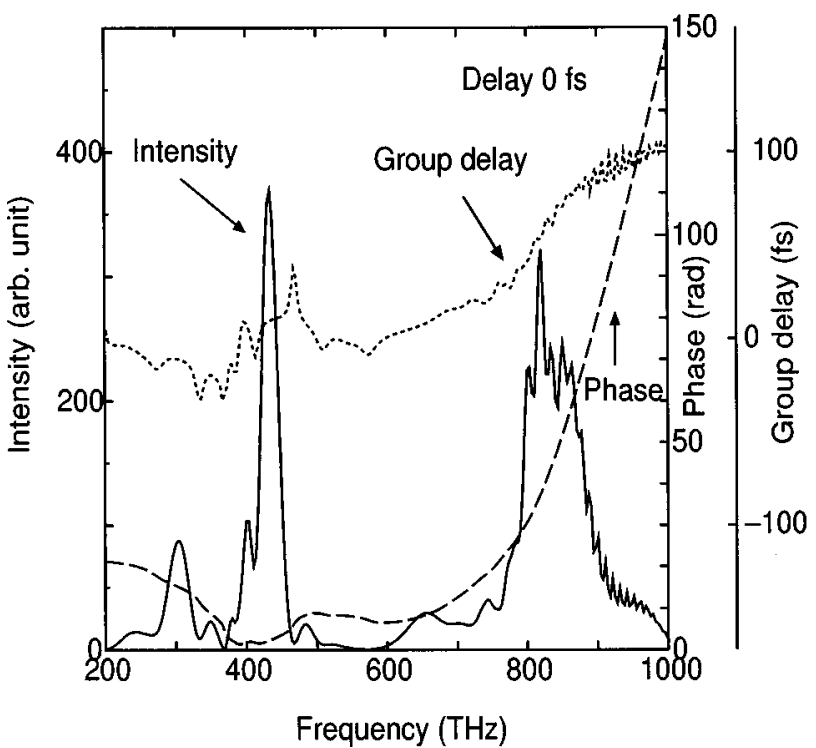

Fig. 3. Calculated intensity, phase, and group delay versus frequency of the fundamental and second-harmonic waves after propagation through an argon-filled capillary fiber under the condition $P_{1}=P_{2}=1.29 \mathrm{GW}, T_{d 2}=0 \mathrm{fs}$.

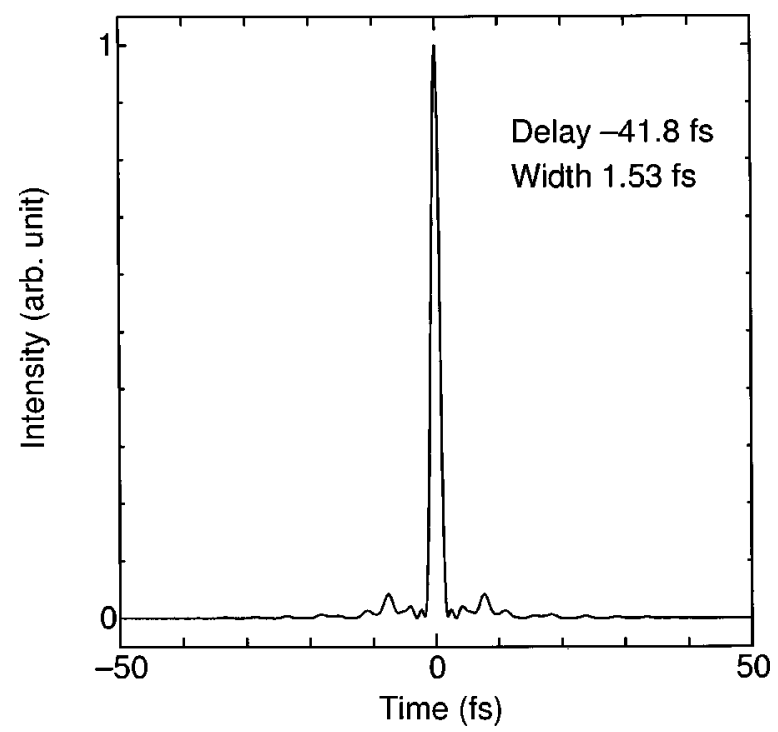

Fig. 4. Calculated intensity versus time of the compressed pulse after its phase is compensated for by the spatial phase modulator. Other parameters are the same as those of Fig. 2.

\section{DISCUSSIONS}

Figure 2 shows that the spectra of fundamental and second-harmonic waves are just overlapping at peak powers of $1.29 \mathrm{GW}$ each, as is expected from the evaluation of the required peak powers in Section 5. By comparing Figs. 2 and 3, we can see the effect of a delay time on the spectrum broadening. For Fig. 2 the delay time is chosen such that both pulses meet near the fiber exit. In this case the IPM effect tends to cover the spectrum fully between fundamental and second-harmonic waves. For Fig. 3, on the other hand, the delay is chosen such that both pulses meet at the fiber entrance. In this case the IPM effect tends to broaden the spectrum in opposite directions, and not much power is left around the center.

The maximum power that does not damage the medium may be determined by use of the self-focusing effect. $^{7}$ For bulk argon, the maximum power may be evaluated to be

$$
P_{c}=\lambda^{2} / 2 \pi n_{2}=\left\{\begin{array}{ll}
3.1 \mathrm{GW} & (\lambda=790 \mathrm{~nm}) \\
0.77 \mathrm{GW} & (\lambda=395 \mathrm{~nm})
\end{array},\right.
$$

which is smaller than the $1 \mathrm{GW}$ of power employed for the second-harmonic wave. However, a recent theoretical estimation has shown that for a hollow waveguide the damage threshold is $\sim 5$ times larger than the bulk medium. ${ }^{20}$ Thus it is likely that pulses with $1.29-\mathrm{GW}$ of peak power can be used without damage problems.

\section{CONCLUSION}

Ultrabroadband optical pulse generation that uses SPM and IPM in a gas-filled hollow waveguide has been analyzed. Analytic results of chirps without dispersion but with loss in the fiber have been derived for the Gaussian input pulses. By use of these equations, the optimum conditions relating to a delay time between two pulses and to input peak powers have been found for fully cover- 
ing the spectrum between $\omega_{1}$ and $\omega_{2}$. As an example, for the experimentally realizable parameters that use the fundamental and the second-harmonic waves from a Ti:sapphire laser-amplifier system in an argon-filled capillary fiber, the optimum delay time and the required pulse peak powers have been obtained. These results have been verified by numerical calculations, including dispersion effects. These calculations show that by this method it is in fact possible to generate an ultrabroadband quasi-linear chirped optical pulse that fully covers the spectrum between these two waves.

This technique for the generation of quasi-linearchirped, white-spectral pulses can be applied to optical pulse monocyclization as well as to the realization of independently synthesized, synchronized multicolor femtosecond beams as a new optical source, such as a femtosecond photon factory. Experimental verification of this theory is under way in our laboratory.

\section{ACKNOWLEDGMENTS}

The authors thank Hiroyasu Sone of Muroran Institute of Technology for providing the nonlinear fiber propagation program that is used in this study with some modifications.

\section{REFERENCES AND NOTES}

1. M. Yamashita, H. Sone, and R. Morita, Jpn. J. Appl. Phys., Part 2 35, L1194 (1996).
2. M. Yamashita, H. Sone, R. Morita, and H. Shigekawa, IEEE J. Quantum Electron. 34, 2145 (1998).

3. A. Baltuška, Z. Wei, M. S. Pshenichnikov, D. A. Wiersma, and R. Szipocs, Appl. Phys. B: Lasers Opt. 65, 175 (1997).

4. A. Baltuška, Z. Wei, M. S. Pshenichnikov, and D. A. Wiersma, Opt. Lett. 22, 102 (1997).

5. H. Nishioka, W. Odajima, K. Ueda, and H. Takuma, Opt. Lett. 20, 2505 (1995).

6. M. Nisoli, S. De Silvestri, and O. Svelto, Appl. Phys. Lett. 68, 2793 (1996).

7. M. Nisoli, S. Stagira, S. De Silvestri, O. Svelto, S. Sartania, Z. Cheng, M. Lenzner, Ch. Spielmann, and F. Krausz, Appl. Phys. B: Lasers Opt. 65, 189 (1997)

8. J. A. Armstrong, N. Bloembergen, J. Ducuing, and P. S. Pershan, Phys. Rev. 127, 1918 (1962).

9. G. D. Boyd and D. A. Kleinman, J. Appl. Phys. 39, 3597 (1968).

10. A. Yariv, Optical Electronics (Saunders, Philadelphia, Pa., 1991).

11. R. Wynands, O. Coste, C. Rembe, and D. Meschede, Opt. Lett. 20, 1095 (1995).

12. G. P. Agrawal, Nonlinear Fiber Optics (Academic, San Diego, Calif., 1989).

13. G. P. Agrawal, P. L. Baldeck, and R. R. Alfano, Phys. Rev. A 40, 5063 (1989).

14. E. A. J. Marcatili and R. A. Schmeltzer, Bell Syst. Tech. J. 43, 1783 (1964).

15. H. J. Lehmeier, W. Leupacher, and A. Penzkofer, Opt. Commun. 56, 67 (1985).

16. A. Dalgarno and A. E. Kingston, Proc. R. Soc. London, Ser. A 259, 424 (1960).

17. A. M. Weiner, D. E. Leaird, J. S. Patel, and J. R. Wullert II, IEEE J. Quantum Electron. 28, 908 (1992).

18. The authors thank a reviewer for pointing out this issue.

19. D. Yelin, D. Meshulach, and Y. Silberberg, Opt. Lett. 22, 1793 (1997).

20. G. Tempea and T. Brabec, Opt. Lett. 23, 762 (1998). 